BULLETIN Bulletin hispanique

HISPANIQUE Université Michel de Montaigne Bordeaux

$115-2$ | 2013

Les traductions vieillissent-elles?

\title{
Le traitement des proverbes dans les traductions du Quichotte
}

\section{Maryse Privat}

\section{(2) OpenEdition}

Journals

\section{Édition électronique}

URL : http://journals.openedition.org/bulletinhispanique/2713

DOI : 10.4000/bulletinhispanique. 2713

ISSN : 1775-3821

\section{Éditeur}

Presses universitaires de Bordeaux

\section{Édition imprimée}

Date de publication : 28 décembre 2013

Pagination : $529-540$

ISBN : 978-2-86781-908-7

ISSN : 0007-4640

Référence électronique

Maryse Privat, "Le traitement des proverbes dans les traductions du Quichotte », Bulletin hispanique [En ligne], 115-2 | 2013, mis en ligne le 14 février 2017, consulté le 19 avril 2019. URL : http:// journals.openedition.org/bulletinhispanique/2713; DOI : 10.4000/bulletinhispanique.2713 


\title{
Le traitement des proverbes dans les traductions du Quichotte
}

\author{
Maryse Privat \\ Universidad de La Laguna
}

Cet article présente une analyse parémiologique et contrastive sur treize proverbes des chapitres 5 et 50 de la deuxième partie du Don Quichotte de Cervantès comparant cinq traductions de ces mêmes proverbes. Cette étude évalue également le degré de prise de conscience du traducteur face aux formes parémiologiques et le degré de réussite de sa version du proverbe en français.

Mots-clés : parémiologie, proverbe, Don Quichotte, traduction.

Este artículo presenta un análisis paremiológico contrastivo de trece refranes sacados de los capitulos 5 y 50 de la segunda parte del Quijote de Cervantes comparando cinco traducciones de estos refranes. Este estudio valora asimismo el grado de concienciación del traductor frente a una paremia y el grado de acierto de su versión del refrán vertido al francés.

Palabras claves : Paremiología, proverbio, Don Quijote, traducción.

This article presents a contrastive analysis of thirteen proverbs of the Quixote of Cervantes in the chapters 5 and 50 (Second part), by comparing them with five translations of this work. This study entails to assess the degree of consciousness of the author in front of a 'paremiologique' structure and the degree of success of his version into French.

Keywords : proverb, Don Quixote, translation.

$J$ e commencerai par un proverbe souvent cité par Walter Benjamin, Einmal ist keinmal ("une fois ne suffit pas »). Transposé à la traduction, il nous donne d'emblée le feu vert et la justification des traductions successives d'un même texte et pourrait devenir à la retraduction ce que Traduttore traditore est à la traduction. 
Souvent le discours sur la nécessité ou non d'une retraduction part d'une contradiction. On ne pense retraduction que pour les grandes æuvres, comme Homère, Shakespeare, Cervantès, et plus récemment Freud, Darwin... Avec la métaphore du vieillissement de la traduction apparaît le désir de renouvellement, inhérent semble-t-il au phénomène : le vieux doit être rajeuni, adapté, renouvelé, modernisé, en tout cas modifié. Il est intéressant de remarquer que ce besoin de renouvellement est exprimé parallèlement à l'expression de l'immortalité précisément des grandes œuvres. On considère que certains classiques «n'ont pas pris une ride» et ont su traverser les siècles en gardant une éternelle jeunesse, que le temps n'ôte rien à leur valeur alors qu'on décide dans le même temps qu'il faut rajeunir leurs traductions qui sont taxées d'obsolètes, caduques, dépassées, désuètes, surannées....

La Vulgate de Saint Jérôme, la Bible de Luther, l'Authorized Version sont de grandes traductions. Mais aussi le Plutarque d'Amyot, les Mille et Une Nuits de Galland, le Shakespeare de Schlegel, l'Antigone de Hölderlin, le Don Quichotte de Tieck, le Paradis Perdu de Milton par Chateaubriand, le Poe de Baudelaire, le Baudelaire de Stefan George : voilà une liste, nullement exhaustive, de grandes traductions. Qui ne vieillissent pas. (Berman, 1990 : 2)

Les belles traductions vieillissent, comme les œuvres, au sens où elles continuent à être actives, à être lues. Même après que l'état de la langue où elles ont été écrites a vieilli. (Meschonnic, 1999 : 22)

Que les traductions ne vieillissent pas ou qu' elles vieillissent, la question est de savoir si ces traductions vieillissent bien... Par ailleurs, les mêmes interrogations reviennent dans les débats et études sur la nécessité ou non d'une retraduction : pourquoi certaines traductions vieillies doivent-elles être renouvelées et d'autres non? Y a-t-il une date de péremption des traductions? Quelle est la position du (re)traducteur par rapport à ceux qui l'ont précédé ? Sa tâche est-elle simplifiée par ses prédécesseurs ou non ? Comment conserver la pérennité de l'écrivain face à l'inconstance du temps et la variabilité de la langue ? Les deux postures décrites par Schleimarcher, rapprocher le texte du lecteur ou le lecteur du texte, sont-elles toujours d'actualité et sont-elles cumulables ? Certains traducteurs suivent simultanément les deux démarches, tel Jean Canavaggio qui, dans ses notes introductives à la nouvelle traduction de 2001 du Quichotte, affirme vouloir " d'une part, rapprocher les textes anciens du lecteur moderne, d'autre part permettre à ce lecteur de remonter vers des faits de langue et des codes qu'il redécouvre ou même découvre pour son plaisir ". (Cervantès, 2001 : LXXV).

Que faire donc de la langue originale de ce texte qui a pris de l'âge ? Jusqu'où mener la modernisation ? Comment procéder pour garder une certaine patine d'ancienneté, respecter la spécificité des textes anciens sans pour autant accumuler les particularités lexicales ou syntaxiques qui ne sont plus les nôtres et pourraient déconcerter le lecteur d'aujourd'hui ? Comment mener à bien ce 
« rajeunissement discret " qu'évoque Canavaggio (Cervantès, 2001 : LXXIV) et, puisque le thème de mon intervention concerne les proverbes, comment rajeunir discrètement les proverbes présents dans l'œuvre de Cervantès?

Les proverbes et le traitement de la traduction des proverbes dans les œuvres littéraires constitue une de mes lignes investigatrices, ce qui m'a amenée tout naturellement à lire ou relire les traductions françaises du Don Quichotte de Cervantès, à l'aune de ses proverbes ${ }^{1}$.

Rappelons la particularité de la traduction des proverbes qui consisteà ménager un aparté spécifique pour l'ensemble des figements, incluant expressions figées et proverbes. Plus que de traduction, il s'agit de rechercher dans la somme parémiologique française l'existence d'un équivalent pré-existant et attesté de façon à pouvoir l'insérer dans la traduction. Plusieurs questions en viennent naturellement et conséquemment à être posées, à savoir : existe-t-il toujours un équivalent français attesté? Si cet équivalent existe, peut-il, de par son lexique, de par la métaphore utilisée le cas échéant, se glisser sans encombre dans le contexte? Au cas où cet équivalent n'existe pas, comment procéder ? Les deux proverbes, en L1 et L2, ont-ils ou avaient-ils la même fréquence d'utilisation ? Ou encore doit-on conserver l'ambigüité ou la non compréhension de certains proverbes? C'est au traducteur que revient la tâche de répondre à ces questions. Encore faut-il que le (re)traducteur se les pose, ce qui implique d'une part qu'il ait reconnu les proverbes dans le texte à traduire et, d'autre part, qu'il les traite (ainsi que les autres expressions figées de la langue) comme des entités à part et non comme de simples éléments du discours.

Pour limiter ici non pas la portée de mes réflexions mais le volume de mes exemples illustratifs, je me suis limitée à cinq retraductions et aux chapitres 5 et 50 de la deuxième partie du Don Quichotte, et ce pour plusieurs raisons. Tout d'abord, ces deux chapitres se font écho, le chapitre 50 étant une réponse, une suite au chapitre 5. Ensuite, il y est question de la condition de la femme, autre sujet qui m'intéresse dans mes recherches parémiologiques. Enfin, ces deux chapitres pourraient être une sorte de paradigme de la représentativité des proverbes dans le Don Quichotte : les proverbes y sont omniprésents, parfois maltraités ou altérés dans la bouche des personnages. La trame narrative de ces deux chapitres justifie la présence de certains thèmes récurrents argumentés par des proverbes illustrant notamment la place des femmes dans la société, le mariage, le conformisme et la mise en garde contre les dangers guettant celui qui voudrait changer de position sociale et, enfin, l'idée selon laquelle il faut saisir l'occasion quand elle se présente.

Examinons comment sont traités les proverbes du Quichotte dans leurs traductions françaises. Pour cette étude, j'ai comparé cinq traductions, depuis celle de Viardot de 1836, la révision-modernisation par Jean Cassou des premières traductions du XvII ${ }^{\mathrm{e}}$ siècle d'Oudin-Rosset, publiée dès 1934

1. Cet article fait suite à une première étude sur la traduction des proverbes du Don Quichotte. Voir Privat, 1999. 
dans La Bibliothèque de La Pléiade, en passant par celle plus moderne d'Aline Schulman, publiée aux éditions du Seuil en octobre $1997^{2}$, et celle de Jean Canavaggio ${ }^{3}$ publiée en 2001 dans la collection «Bibliothèque de la Pléiade " qui décidait ainsi de refaire une nouvelle traduction du Quichotte, pour enfin parvenir à la dernière traduction, celle de Jean-Raymond Fanlo publiée en 2008 dans La Pochothèque.

Il est intéressant d'observer la ou les différences d'approche des cinq (re) traducteurs face à la traduction des mêmes proverbes.

Pour le premier, Viva la gallina aunque sea con su pepita, tous les traducteurs donnent une version littérale du proverbe Vive la poule même avec sa pépie [eûtelle la pépie]. Seule Schulman opte pour une solution autre, en rendant ce proverbe, non attesté en français, par l'expression «Le remède serait pire que le mal ». Bien que cette expression semble éloignée du proverbe original, elle correspond parfaitement à la situation où Teresa répond à Sancho qui se dit prêt à tomber raide mort s'il ne peut devenir Gouverneur. Néanmoins, si l'expression convient dans le contexte et s'insère dans la philosophie de la traductrice d'utiliser un langage clair et adapté à un public moderne, on perd le jeu entre 'mourir / vivre', essentiel dans la réplique de Teresa où le mot vivre apparaît quatre fois et l'allusion à la mort deux fois. De plus, la couleur proverbiale disparaît : plus d'image, plus de métaphore, plus de rime. Peut-être aurait-on pu penser au proverbe français Chien en vie vaut mieux que lion mort, où la métaphore animale est conservée, ainsi que l'alternance vie / mort.

Le deuxième proverbe La mejor salsa del mundo es la hambre est traduit unanimement La meilleure sauce du monde, c'est la faim, traduction littérale parfaitement adaptée, comme l'auraient été aussi ces proverbes existant en français sur le même thème : À qui a faim tout est pain ou Il n'est sauce que d'appétit, ce dernier s'éloignant quelque peu du contexte, l'appétit et la faim étant deux réalités différentes.

Le proverbe $n^{\circ} 3$, Quien te cubre te descubre, ne présente pas de problème particulier pour la traduction, "couvre " et " découvre " gardant la même rime qu'en espagnol. C'est pourquoi les cinq traductions optent pour une traduction littérale, Qui te couvre te découvre, parémie relevée dans les recueils français comme "proverbe de Cervantès " ainsi que sa variante Tout ce qui couvre découvre. Si Cervantès avait opté pour mettre dans la bouche de Teresa un proverbe plus original, plus haut en couleur, plus pittoresque, on aurait pu choisir parmi les proverbes français exprimant cette même idée Il n'est d'orgueil que de sot revêtu ou encore Plus haut monte le singe, plus il montre son cul.

Le proverbe $\mathrm{n}^{\circ} 4, Y$ ándeme yo caliente, y ríase la gente, parmi les proverbes espagnols les plus connus, n'a pas d'équivalent français correspondant à un même degré de fréquence. Le proverbe Pourvu que j’aie chaud, des rires peu

2. Réédité en juin 2009 dans la collection Points.

3. La traduction porte la triple signature de Claude Allaigre, Jean Canavaggio et Michel Moner. 
me chaut est acceptable dans une traduction qui se veut respectueuse de la langue originale, ce verbe chaloir conservant l'archaïsme recherché et la rime chaud / chaut correspondant à caliente / gente, tout comme la traduction de Viardot, Pourvu que jaille les pieds chands, je laisse rire les badauds qui respecte la rime caliente / gente par l'assonance chauds / badauds. Pour ce qui est de la traduction de Schulman, là encore, l'adaptation choisit délibérément une expression courante et actuelle, parfaitement appropriée au contexte : Là où il $y$ a de la gêne, il n'y a pas de plaisir, même si la distance semble apparemment grande entre les deux parémies de L1 et L2.

Il en va de même pour le proverbe $n^{\circ} 5$, Quien te da el hueso no te querría ver muerto. Aucun proverbe français attesté ne reprend, à ma connaissance, cette idée de 'Ce n'est pas grand-chose mais le cœur y est'. C'est pourquoi la traduction de 1997 de Schulman me semble convenir à nouveau: Comme on dit, l'intention y est, l'introduction " comme on dit ", absente du texte original, servant à marquer le caractère figé de l'expression utilisée. Quant aux traductions littérales des autres versions étudiées, elles ont l'avantage de l'expressivité originale espagnole, de par la conservation littérale de la métaphore du chien et de l'os.

Pour continuer avec les proverbes pour lesquels il n'existe pas d'équivalent attesté en français si ce n'est justement la parémie cervantine, voici un autre exemple mettant en scène la femme. Le proverbe $\mathrm{n}^{\circ} 6$, Mejor parece la hija mal casada que bien abarraganada, est donné en français dans sa traduction littérale. Seules quelques variantes lexicales les distinguent. Si mal mariée fait presque l'unanimité de façon justifiée, on constate une plus grande variété face au vocable abarraganada qui se décline à travers les adjectifs amourachée, entretenue, acoquinée ou encore bon concubinage et [en puissance de] bon amant.

Les deux proverbes suivants illustrent la place revenant à la femme dans la société, La mujer honrada, la pierna quebrada y en casa (7) et La doncella honesta, el hacer algo es su fiesta (8). Le premier est rendu littéralement dans quatre traductions qui conservent cette métaphore insolite de la jambe cassée ne faisant référence à aucun proverbe français, contrairement à la parémie espagnole, très largement répandue et reconnue d'emblée comme proverbiale. Il en est de même pour le second, rendu simplement mot à mot dans les mêmes quatre versions et gardant la rime grâce à la similitude des deux langues (honesta / fiesta et honnête / fête). Dans ces deux cas, la traductrice Aline Schulman se distingue et cherche une cohérence textuelle en accord avec les objectifs fixés pour sa traduction en essayant, non pas de préserver l'original espagnol, mais de trouver dans la somme des proverbes français consacrés aux femmes deux formulations appropriées de par leur contenu sémantique et lexical. Fille cachée, fille cherchée et Filles et poules se perdent de trop courir reprennent l'idée exprimée dans le texte original, où le contenu exact de la forme parémique n'est pas d'une importance primordiale, tout autre proverbe sur les femmes et leur rôle et place dans la maison ayant fait l'affaire. De plus, les proverbes choisis, attestés en français, gardent une syntaxe et un lexique adaptés à sa volonté de modernité relative, ce que n'auraient pas fait d'autres proverbes français 
attestés comme Fille qui trotte et géline qui vole de légier sont adirées ou Fille oisive a mal pensive, fille trop en rue tost perdue, d'emblée rejetés de par leur langue trop archaïsante.

Il en va de même pour le proverbe suivant, Al hijo de tu vecino, limpiale las narices y métele en tu casa (9), traduit unilatéralement et littéralement dans toutes les traductions, sauf dans la version modernisée de 1997 qui, là encore, cherche et trouve un équivalent français attesté, tout à fait adapté, Qui loin va se marier on veut tromper, même si le proverbe exact et complet devrait être Qui loin va se marier sera trompé ou veut tromper. Cet autre proverbe français plus imagé aurait également été tout aussi satisfaisant: Où la chèvre est attachée, il faut qu'elle broute.

Quant au proverbe $\mathrm{n}^{\circ} 10$, Tal el tiempo tal el tiento, les traducteurs offrent des versions variées. Les deux premiers proposent Selon le temps le bâton, Tel le temps, tel le traitement alors que dans un autre chapitre (II, 54), pour ce même proverbe, ils suggèrent respectivement Tel le temps, telle la conduite et Tel le temps, tel le soin, ce qui montre qu'ils ne s'attachent pas systématiquement à la cohérence dans la traduction des parémies présentes dans l'ensemble du texte de Cervantès. Les deux (re)traducteurs plus récents proposent Autres temps autres mours et Il est un temps pour tout, s'éloignant quelque peu du sens de la formule parémique Tal el tiempo tal el tiento qui signifie qu'il faut prendre le temps comme il vient et s'adapter aux changements ou, comme dit Sancho dans le chapitre 5, lorsqu'il se refuse à parler en proverbes et qu'il dit : "Dejémonos llevar deste viento favorable que nos sopla ». On aurait pu également penser au proverbe français $\grave{A}$ tel saint, telle offrande ou Selon le temps la voile. Quant à Schulman, elle renonce à ces proverbes pour choisir une expression non proverbiale mais authentique et courante Il faut prendre le temps comme il vient. Précisons que la parémie attestée complète serait la suivante: Prenez le temps comme il vient, le vent comme il souffle, la femme comme elle est.

Un autre proverbe exprime cette même idée de "saisir l'occasion sans chercher à comprendre »: Cuando te dieren la vaquilla, corre con soguilla (11). Les équivalents français, Il faut saisir l'occasion par les cheveux ou L'occasion n'a qu'un cheveu, L'occasion est chauve, sont certes bien fades face à la métaphore de la vache et de la corde ${ }^{4}$, justifiant assurément la décision de quatre traducteurs de garder l'image du proverbe original, hésitant entre veau, génisse et vachette ou corde, longe et cordelette, le dernier traducteur ayant voulu conserver la rime existante en espagnol vaquilla / soguilla par vachette / cordelette. Ce proverbe n'est traduit par un équivalent français que par Schulman, Un tiens vaut mieux que deux tu l'auras. Bien que ce proverbe soit acceptable en contexte, on peut objecter qu'il traduit plus la garantie incontestable de la réalité présente face à un futur hypothétique alors que l'idée du proverbe espagnol illustre la nécessité de saisir l'occasion quand elle se présente, sans allusion au futur.

4. Ce proverbe non attesté en français existe en anglais: When they give a cow, run and fetch a haller. 
Je finirai cet échantillonnage proverbial par une facette toute particulière mais courante de la parémiologie, qui est l'altération occasionnelle des proverbes, malmenés dans la bouche des protagonistes, par jeu ou par dérision. L'altération de proverbes inclut aussi bien la déformation plus ou moins involontaire (proverbe $\mathrm{n}^{0} 12$ ) que le troncage souvent délibéré (proverbe $\mathrm{n}^{\circ} 13$ ). Cette modification des proverbes repose sur une bonne connaissance de ces mêmes proverbes, car jouer sur la déformation suppose la connaissance préalable de ce même proverbe de la part du public lecteur. Il en va de même pour les proverbes tronqués : pour qu'un proverbe puisse être amputé de sa fin, il faut qu'il soit suffisamment connu donc reconnaissable. La chute de la phrase est évidente pour qui connaît le proverbe dans son entier. Mais ne reconnaîtra que celui qui connait : $\grave{A}$ bon entendeur..., Pierre qui roule..., Un tiens vaut mieux...

Le premier cas d'altération est illustré par Teresa qui affirme: Allá van reyes do quieren leyes alors que le proverbe espagnol original dit l'inverse, Allá van leyes do quieren reyes (les lois vont là où le veulent les rois). Dans la première traduction, Viardot rétablit inexplicablement sa formulation exacte, produisant ainsi un contresens de traduction puisque le proverbe est volontairement estropié dans l'original espagnol pour montrer l'ignorance de celle qui le prononce. Quant à la traduction de Schulman, qui propose La loi est la loi, même pour les rois, elle est partiellement à rejeter puisque, bien que gardant le sens que veut lui donner Teresa dans le texte de Cervantès, cette phrase ne restitue pas un proverbe déformé. Ici, le dicton juridique français Que veut le roi le veut la loi ou sa variante Si veut le roi, si veut la loi aurait été pleinement satisfaisant dans leur version déformée volontaire Que veut la loi le veut le roi ou Si veut la loi, si veut le roi.

Après cet exemple de proverbe déformé, vient un proverbe tronqué: Viose el perro en bragas de cerro (13). À ce début, tout contemporain de Cervantès saurait ajouter la fin : [...] y no conoció a su compañero ou sa variante : [... y él fiero que fiero. Observant les traductions proposées, on retrouve la volonté de Viardot, face aux proverbes tronqués, de rétablir systématiquement le proverbe dans son intégralité. La traduction de 2001 de Allaigre, Canavaggio et Moner se risque à une adaptation, et non une traduction, plus ou moins littéraire en choisissant ce vers de La Fontaine, Le geai s'est paré des plumes du paon, gardant tout à la fois le parallélisme animalier et le sens de " chercher à tirer parti, se vanter d'avantages qui ne nous correspondent pas ». Quel proverbe français de sens similaire, assez connu pour être tronqué, aurait pu être utilisé ici ? Aucun à ma connaissance. Le proverbe Vilain enrichi ne connâ̂t ni parent ni ami ne saurait être tronqué sans risquer de devenir une complète énigme pour le lecteur. La traduction littérale du proverbe espagnol sous sa forme tronquée pourrait être incomprise, ce qui explique que Viardot opte pour une traduction littérale du proverbe complet, licence cette fois toute justifiée. Deux autres traducteurs choisissent la littéralité : Le chien se vit en culotte de soie / velours où l'opposition sémantique chien /soie-velours laisse supposer que le chien change de condition sociale, laissant deviner au lecteur néophyte qu'il s'agit d'un 
proverbe persiflant les parvenus. Quant à la traduction de 1997, elle choisit une troisième formule s'éloignant de l'original tout en gardant l'impact de la réplique de Sanchica, et réunissant une expression populaire et un proverbe enchaînés dans la même phrase : si quelquiun [...] vient me dire qu'on n'a pas gardé les oies ensemble et que l'habit ne fait pas le moine.

Après examen de ces proverbes et de leurs différentes traductions, on peut entrevoir l'attitude et la démarche globale de chaque traducteur face aux proverbes. Nous ne pouvons que constater que les deux premiers (re) traducteurs, Viardot et Cassou, ne semblent pas considérer les proverbes comme des unités langagières propres, ne semblent pas percevoir la nécessité d'un traitement spécifique pour ces formules figées que sont les proverbes et proposent systématiquement une simple traduction littérale du proverbe espagnol, qu'un équivalent français existe ou non. Quant aux deux retraductions les plus récentes, on perçoit une plus grande cohérence ou conscience parémiologique, notamment dans le cas de Jean-Raymond Fanlo qui cherche à restituer la couleur du proverbe en choisissant pour sa traduction littérale un vocable permettant de conserver pour le moins la rime du proverbe, comme dans l'original espagnol. Ainsi le choix de foyer au lieu de maison dans Femme respectée, jambe cassée, et au foyer, le choix de acoquinée qui rime avec mariée dans Il vaut mieux une fille mal mariée que bien acoquinée, et enfin vachette et cordelette qui respectent mieux la rime espagnole que le veau ou la génisse des autres traductions littérales: Si on te donne la vachette, cours à la cordelette.

Quant à la traduction d'Aline Schulman, elle suit une approche autre. Contrairement aux autres (re)traducteurs, on peut affirmer qu'elle voit les proverbes et les traite différemment. Son parti-pris de (re)traductrice est celui d'une rhétorique de l'oralité et l'objectif qu'elle s'est clairement fixé est " de restituer la modernité du Quichotte dans sa totalité, c'est-à-dire aussi, et surtout, dans la modernité de sa langue ", (Cervantès, 1997 : 15).

Comment restituer la modernité des proverbes alors qu'ils conservent souvent un aspect archaïque, dû à la syntaxe, au lexique mais également au fait que ce sont des formes figées auxquelles le public en général n’a plus recours? Ce n'est pas un hasard si les seuls proverbes qui ont traversé les siècles pour parvenir jusqu'à nous soient des proverbes à forme simple : Pierre qui roule n'amasse pas mousse, Qui veut voyager loin ménage sa monture, Un tiens vaut mieux que deux tu l'auras, Qui sassemble se ressemble, Qui trop embrasse mal étreint... D'autre part, ces formes archaïsantes étant fixes, figées, comment pourrait-on les moderniser ? Il semblerait qu'il y ait là une contradiction, mais cette impossibilité de départ n'est que relative car de nombreux proverbes ont non seulement des variantes mais se déclinent sous plusieurs formes, chacune dans sa forme figée. Pour illustrer une même idée, cohabitent parfois plusieurs proverbes. Ainsi, par exemple, pour dire qu'il faut agir sans attendre dès que l'occasion se présente, on trouve divers proverbes: Il faut puiser quand la corde est au puits, Il faut battre le fer tant qu'il est chaud, Quand on tient la poule, il faut la plumer ou encore Il faut écorcher l'anguille quand on la tient. 
Dans la préface de la traduction de Schulman, Jean-Claude Chevalier ajoute, en ce qui concerne la traduction des proverbes, que « comme Sancho enfilait des proverbes vrais, inventés ou estropiés, on prendra des libertés avec ses propres proverbes " (Cervantès, 1997 : 13). Cette liberté, toute relative, consiste à agir comme devrait en fait agir tout traducteur conscient des différents aspects de la traduction, y compris des proverbes et formes figées, à savoir : chercher l'équivalent existant qui aura le même contenu sémantique et le même impact, et essayer à défaut d'équivalent, d'y pallier en utilisant une locution ou une expression idiomatique appropriée ou de restituer dans une traduction littérale une miette du rythme et de la couleur de l'original.

"Faut-il mettre les vieux hidalgos en costume de ville ? ", demandait JeanRaymond Fanlo (Cervantès : 2008, 94). Si l'on veut tenir compte du lecteur, ou mieux encore, des lecteurs potentiels, on devrait offrir plusieurs lectures : pour un lecteur érudit ou profane, un public universitaire ou non universitaire, connaisseur ou non connaisseur de la langue du XviI ${ }^{\mathrm{e}}$ siècle et cependant curieux et amateur de littérature espagnole. Et afin de mener une sensibilisationéducation à l'acte de traduire lié à l'acte de lecture, il nous faudrait offrir simultanément diverses traductions d'une même œuvre, tout comme l'on offre plusieurs interprétations d'une même partition musicale. On parle par exemple du pianiste Glenn Gould comme l'" archilecteur " des Variations Goldberg de Bach et, dans le domaine de la traduction, Edith Grossman, traductrice du Don Quijote en anglais en 2003, a été qualifiée par Harold Bloom du «Glenn Gould de la traduction ». En ce qui concerne la lecture et donc la (re)traduction d'œuvres étrangères à offrir aux lecteurs, je répondrai à la question de Fanlo en ces termes : vieil hidalgo ET costume de ville.

Liste des 13 proverbes étudiés (chapitres II, 5 et II, 50)

1. Viva la gallina aunque sea con su pepita.

Vive la poule, eût-elle la pépie. ORC

Vive la poule, même avec sa pépie. $\mathrm{V}$

Le remède serait pire que le mal. $S$

Vive la poule, même avec sa pépie. ACM

Que la poule vive, même avec la pépie. F

\section{La mejor salsa del mundo es la hambre.}

La meilleure sauce du monde, c'est la faim. ORC

La meilleure sauce du monde, c'est la faim. V

La meilleure sauce du monde, c'est la faim. S

La meilleure sauce du monde est la faim. ACM

La meilleure sauce du monde, c'est la faim. F

5. Les initiales utilisées correspondent à celles des traducteurs par ordre chronologique : ORC = Oudin et Rosset revue par Cassou, $\mathrm{V}=$ Viardot, $\mathrm{S}=$ Schulman, ACM = Allaigre, Canavaggio et Moner, $\mathrm{F}=$ Fanlo. 
3. Quien te cubre te descubre.

Qui te couvre et découvre. (sic) ORC

Qui te couvre te découvre. V

Qui te couvre te découvre. S

Qui te couvre te découvre. ACM

Qui te couvre te découvre. F

4. $Y$ ándeme yo caliente, y ríase la gente.

S'en rie qui voudra, pourvu que j'aie les pieds au chaud. ORC

Pourvu que j'aille pieds chauds, je laisse rire les badauds. V

Là où il y a de la gêne, il n'y a pas de plaisir. $S$

Pourvu que j'aie les pieds au chaud, les gens peuvent bien en rire. ACM

Mettez-moi bien au chaud et laissez rire les badauds. F

5. Quien te da el hueso, no te querría ver muerta.

Qui partage avec toi un os ne te voudrait voir morte. ORC

Qui te donne un os ne veut pas ta mort. V

Comme on dit, l'intention y est. S

Qui te donne un os ne veut pas ta mort. ACM

Qui te donne un os ne veut pas ta mort. F

\section{Mejor parece la hija mal casada que bien abarraganada.}

Il vaut mieux voir sa fille en puissance de mauvais mari que de bon amant. ORC

Mieux sied la fille mal mariée que bien amourachée. V

Mieux vaut voir sa fille mal mariée que richement entretenue. $S$

Pour une fille, mieux vaut mauvais mariage que bon concubinage. ACM

Il vaut mieux une fille mal mariée que bien acoquinée. F

\section{La mujer honrada, la pierna quebrada, y en casa.}

Femme de bonne renommée, jambe cassée, et à la maison. ORC

La femme de bon renom, jambe cassée et à la maison. $\mathrm{V}$

Fille cachée, fille cherchée. $S$

Femme de bon renom, jambe cassée et à la maison. ACM

Femme respectée, jambe cassée, et au foyer. F

\section{La doncella honesta, el hacer algo es su fiesta.}

La fille honnête de travailler se fait fête. ORC

La fille honnête, de travailler se fait fête. $\mathrm{V}$

Filles et poules se perdent de trop courir. $S$

La fille honnête, de travailler se fait fête. ACM

Jeune fille honnête, le travail est sa fête. F

\section{Al hijo de tu vecino, limpiale las narices y métele en tu casa.}

Lenfant de ton voisin, mouche-lui le nez et le mets en ta maison. ORC

Au fils de ton voisin, lave-lui le nez et prends-le pour tien. $\mathrm{V}$

Qui loin va se marier, on veut tromper. S

Le fils de ton voisin, mouche-lui le nez et prends-le dans ta maison. ACM

Prends le fils de ton voisin, mouche-le et mets-le dans ta maison. F 
10. Tal el tiempo tal el tiento.

Selon le temps le bâton. ORC

Tel le temps, tel le traitement ! V

Il faut prendre le temps comme il vient. $S$

Autres temps autres mœurs. ACM

Il est un temps pour tout. $\mathrm{F}$

11. Cuando te dieren la vaquilla, corre con soguilla.

Quand on te donnera une génisse, cours-y avec la corde. ORC

Quand on te donnera la génisse, mets-lui la corde au cou. V

Un tiens vaut mieux que deux tu l'auras. $S$

Si on te donne le veau, accours avec la longe. ACM

$\mathrm{Si}$ on te donne la vachette, cours à la cordelette. F

12. Allá van reyes (do quieren leyes).

Les rois font comme les lois. ORC

Ainsi se font les lois comme le veulent les rois. $\mathrm{V}$

La loi est la loi, même pour les rois. $S$

Ainsi sont les rois, comme le veut la loi. ACM

S'en vont les rois où le veulent les lois. $\mathrm{F}$

13. Viose el perro en bragas de cerro...

Le chien se vit en culottes de soie... ORC

Le chien s'est vu en culottes de lin, et il n'a plus connu son compagnon. $\mathrm{V}$

...qu'on n'a pas gardé les oies ensemble et que l'habit ne fait pas le moine. $S$

Le geai s'est paré des plumes du paon... ACM

Le chien s'est vu en culottes de velours... F

\section{Bibliographie}

Bloom Harold (ed.), Cervantes's Don Quixote. Modern Critical Interpretations, Philadelphia, Chelsea House Publishers, 2001.

Cervantès Miguel de, Don Quichotte, Paris, Librairie Générale Française, "La Pochothèque ", 2008. [Traduction, présentation et édition de Jean-Raymond Fanlo].

- Don Quijote de la Mancha, Barcelona, Instituto Cervantes, "Crítica ", 1998. [Édition de l'Instituto Cervantes, Francisco Rico dir.]

- L'ingénieux Hidalgo Don Quichotte de la Manche, Paris, Éditions du Seuil, 1997. [Traduction d'Aline Schulman].

- Euvres romanesques complètes. I, Don Quichotte précédé de La Galatée, Paris, NRF, Gallimard, "Bibliothèque de la Pléiade », 2001. [Traduction de Claude Allaigre, Jean Canavaggio et Michel Moner]

Cervantès Saavedra Miguel de, Don Quichotte, Paris, NRF, Gallimard, "Bibliothèque de la Pléiade », 1949. [Traduction de César Oudin et François Rosset, revue, corrigée et annotée par Jean Cassou] 
— Don Quijote de la Mancha, Madrid, Cátedra, "Letras hispánicas », 1994. [Édition de John Jay Allen].

- L'ingénieux Hidalgo Don Quichotte de la Manche, Paris, J.-J. Dubochet et Cie, 1836. [traduit et annoté par Louis Viardot, tome premier]

- L’ingénieux Hidalgo Don Quichotte de la Manche, Paris, J.-J. Dubochet et Cie, 1836. [traduit et annoté par Louis Viardot, tome deuxième]

Privat Maryse, "Quelques proverbes du Don Quijote vus dans trois traductions françaises», Paremia, 1999, 8, p. 423-428.

Schulman Aline, "Retraduire Don Quichotte», Cahiers internationaux de symbolisme, 1999, 92-94, p. 159-167. 